\title{
Synthetic studies towards the novel diterpenoid rameswaralide: RCM mediated acquisition of the tricyclic core
}

\author{
Goverdhan Mehta* and Sripada Lakshminath \\ Department of Organic Chemistry, Indian Institute of Science, Bangalore 560012, India \\ Received 22 September 2005; revised 25 October 2005; accepted 3 November 2005 \\ Available online 18 November 2005
}

\begin{abstract}
A synthetic approach towards the novel anti-inflammatory diterpenoid rameswaralide from the cis-Corey lactone involving a tandem RCM and Diels-Alder reaction has been conceived and endeavors so far have led to the acquisition of the BCD ring fragment of the natural product.

(C) 2005 Elsevier Ltd. All rights reserved.
\end{abstract}

In 1998, collaborative efforts between the research groups of Venkateswarlu and Faulkner led to the isolation of a novel diterpenoid, rameswaralide 1, from the soft coral Sinularia dissecta collected from the Mandapam coast two years earlier. ${ }^{1}$ The stereostructure of $\mathbf{1}$ was determined through recourse to incisive 2D NMR techniques and chemical transformation to dihydrorameswaralide 2 to reveal the presence of the unusually stable enol moiety. ${ }^{1}$ The unique framework of rameswaralide 1 has close biosynthetic kinship with the diterpenoids mandapamate $\mathbf{3}$ and isomandapamate $\mathbf{4}$ with which it co-occurs in the same soft coral species. ${ }^{2}$ The tetracyclic framework of rameswaralide $\mathbf{1}$, composed of a 5,7,6-fused tricarbocyclic core and functionalities spread in all its rings and embellished with six stereogenic centres is a challenging synthetic target in its own right. However, it is the observation of promising anti-inflammatory activity ${ }^{3}$ associated with $\mathbf{1}$ and its derivatives, with inhibitory activity against TNF- $\alpha$, IL15 , IL-5 and $\mathrm{Cox}_{2}$ with an $\mathrm{IC}_{50}$ in $0.5-5 \mu \mathrm{g} / \mathrm{mL}$ concentration $^{3}$ that has added special attraction to its synthetic pursuit. Although a total synthesis of $\mathbf{1}$ has not been achieved so far, a related model study ${ }^{4}$ has recently surfaced in the literature. As part of our continuing interest in the total synthesis of diterpenoids with 5,7,6-fused tricarbocyclic frameworks, ${ }^{5}$ we disclose in this letter a synthetic approach to $\mathbf{1}$ that has so far culminated in the acquisition of the BCD tricyclic core present in the natural product.

\footnotetext{
*Corresponding author. Tel.: +91 80 22932850; fax: +91 80 23600283; e-mail: gm@orgchem.iisc.ernet.in
}

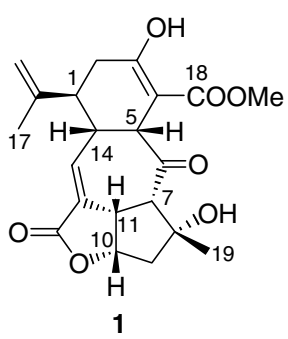

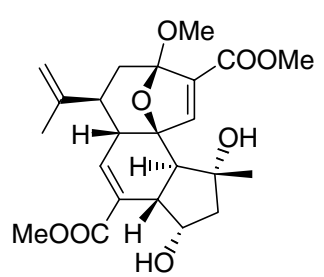

3

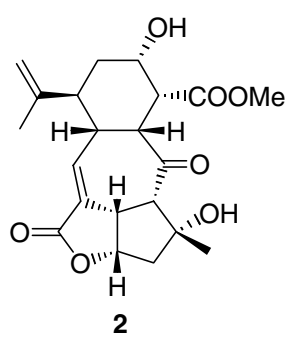

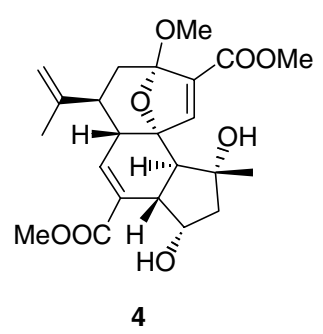

Our synthetic strategy towards $\mathbf{1}$ evolved around the retrosynthetic theme is depicted in Scheme 1. It was envisaged that the cis-fused six-membered ring A could be appended to the preformed tricyclic BCD core $\mathbf{5}$ through [4+2]-cycloaddition protocols on the enone moiety. Thermal and Lewis acid catalyzed Diels-Alder reactions to cycloheptenones are well precedented ${ }^{6}$ and in the case of $\mathbf{5}$ an examination of models revealed that the cycloaddition was expected to be face selective with the diene approaching from the $\alpha$-face to deliver the desired C5,C14 ring junction stereochemistry. The key cycloheptenone moiety in $\mathbf{5}$ was sought to be generated 

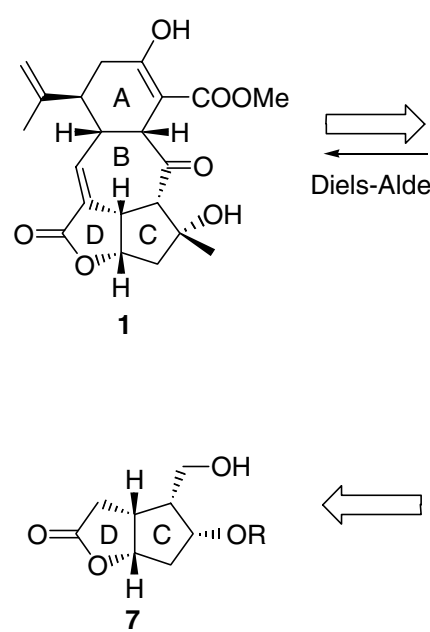

cis-Corey lactone
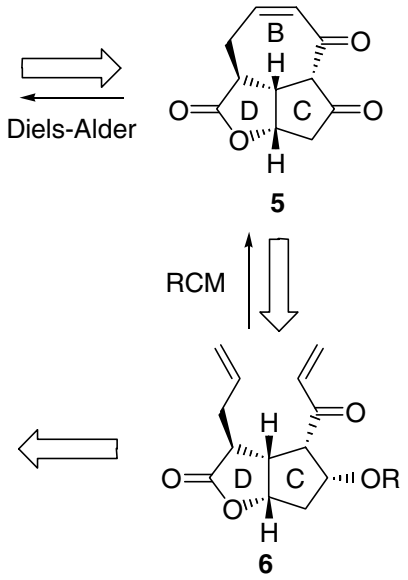

Scheme 1. Retrosynthetic strategy.

through a RCM reaction in $\mathbf{6}$ which, in turn, could be elaborated from the all cis-Corey lactone $7^{7}$ (Scheme 1). The choice of the all cis-Corey lactone 7 as the launch pad was crucial as it embodies the CD rings of the natural product 1 and the required stereochemistry at C7, C10 and C11 is embedded into it. Therefore, our initial objective was to devise a convenient access to 7 in an appropriately protected form for further elaboration.

Several syntheses of the all cis-Corey lactone, emanating from diverse starting materials, have been recorded in the literature, particularly in the context of accessing isoprostanes and related prostaglandin analogues. ${ }^{7}$ However, our present endeavors warranted development of a de novo synthesis of 7 and that became our initial objective. Towards this end, the recently reported ${ }^{8}$ and readily available 7-keto-norbornene derivative 8 was selected as the starting material. Wittig methoxymethylenation in $\mathbf{8}$ led to enol ether $\mathbf{9}$ and carefully controlled acid catalyzed hydrolysis $\left(<0{ }^{\circ} \mathrm{C}\right)$ in 9 to the intermediate aldehyde $\mathbf{1 0}$ followed by immediate borohydride reduction led to a readily separable mixture (4:1) of the $s y n-11^{9}$ and anti-12 alcohols in good yield (Scheme 2). Protection of the hydroxyl group in $\mathbf{1 1}$ as its TBS derivative followed by acetate hydrolysis and TPAP oxidation furnished the syn-norbornenone derivative $13 .{ }^{9}$

Baeyer-Villiger oxidation of $\mathbf{1 3}$ presented some initial chemoselectivity problems (Scheme 3) because of the steric shielding of the C2 carbonyl group by the C7 syn substituent but high chemoselectivity was secured by carrying out the reaction in basic $\mathrm{H}_{2} \mathrm{O}_{2}$ to obtain lactone $\mathbf{1 5}$ with the complete exclusion of the epoxide product 14. Reconstructive iodolactonization ${ }^{10}$ of $\mathbf{1 5}$ furnished iodolactone $16^{9}$ in good yield, however, we were surprised to find that the TBS protection was lost during the process (Scheme 3). The two hydroxyl groups in $\mathbf{1 6}$ were protected as the TES derivative $\mathbf{1 7}$ and reductive deiodination furnished the all cis-Corey lactone derivative 18 (Scheme 3). ${ }^{9}$ Although the route to 18 was somewhat long, its execution was straightforward and this key intermediate could be routinely obtained in gram quantities.

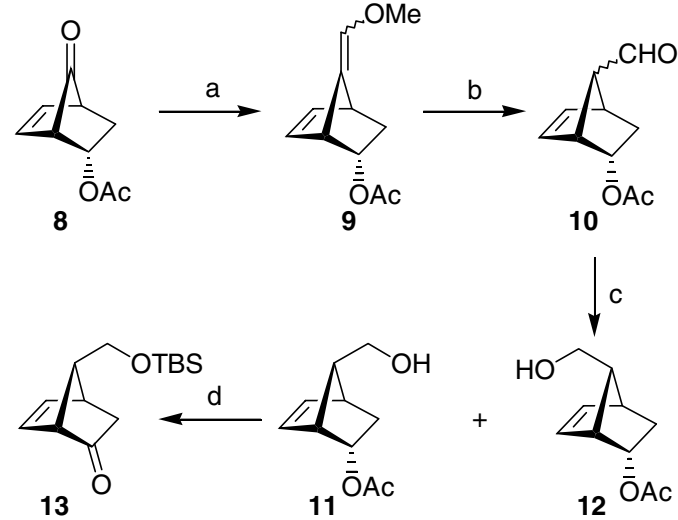

Scheme 2. Reagents and conditions: (a) $\mathrm{CH}_{3} \mathrm{OCH}_{2} \mathrm{PPh}_{3} \mathrm{Cl}, \mathrm{KO}^{t} \mathrm{Am}$, THF, $0{ }^{\circ} \mathrm{C}, 2 \mathrm{~h}, 80 \%$; (b) $\mathrm{HClO}_{4}$ (cat.), DCM, $0{ }^{\circ} \mathrm{C}, 2 \mathrm{~h}, 93 \%$; (c) $\mathrm{NaBH}_{4}, \mathrm{MeOH}, 0{ }^{\circ} \mathrm{C}, 1 \mathrm{~h}$, quant., 11:12=4:1; (d) (i) $\mathrm{TBSCl}$, imidazole, DCM, rt, $1 \mathrm{~h}, 96 \%$; (ii) $\mathrm{K}_{2} \mathrm{CO}_{3}, \mathrm{MeOH}, 10 \mathrm{~h}, 92 \%$; (c) TPAP, NMMO, rt, DCM, 3 h, 94\%.

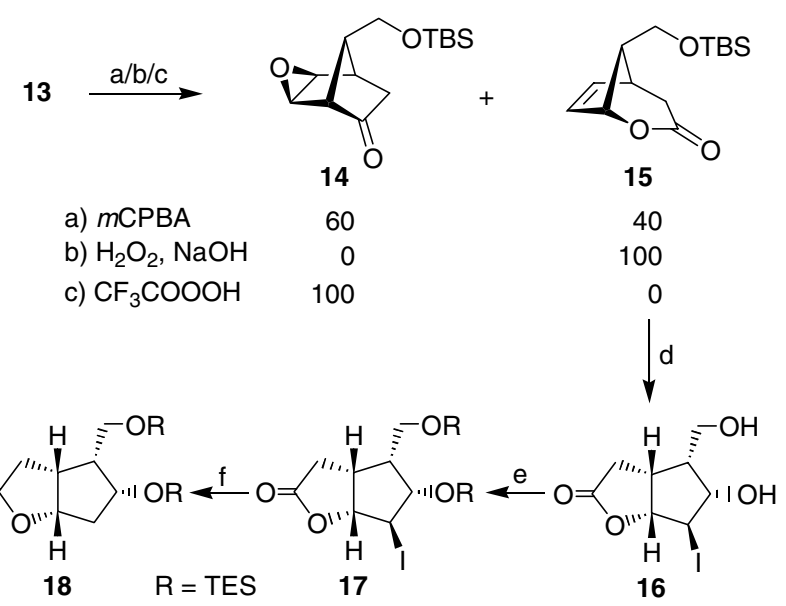

Scheme 3. Reagents and conditions: (a) $m \mathrm{CPBA}, \mathrm{NaHCO}_{3} / \mathrm{PTSA}$, $0{ }^{\circ} \mathrm{C}$ to rt, $2 \mathrm{~h}, 89 \%$; (b) $\mathrm{H}_{2} \mathrm{O}_{2}, \mathrm{NaOH}, \mathrm{MeOH}, 0{ }^{\circ} \mathrm{C}, 2 \mathrm{~h}, 95 \%$; (c) $\mathrm{CF}_{3} \mathrm{COOOH}, \mathrm{KH}_{2} \mathrm{PO}_{4}, \mathrm{DCM}, 0{ }^{\circ} \mathrm{C}, 1 \mathrm{~h}, 95 \%$; (d) (i) $\mathrm{NaOH}, \mathrm{MeOH}$, rt, 12 h; (ii) KI, $\mathrm{I}_{2}, \mathrm{H}_{2} \mathrm{O}, \mathrm{rt}, 3 \mathrm{~h}, 86 \%$ (two steps); (e) TESCl, Py, rt, 12 h, $90 \%$; (f) $\left({ }^{n} \mathrm{Bu}\right)_{3} \mathrm{SnH}, \mathrm{AIBN}, \mathrm{C}_{6} \mathrm{H}_{6}, \Delta, 2 \mathrm{~h}, 95 \%$.

The next task was to set up the RCM reaction to generate the seven-membered $B$ ring of rameswaralide $\mathbf{1}$. In this context, lactone $\mathbf{1 8}$ was stereoselectively allylated to yield 19 with the addition exclusively from the convex face (Scheme 4). ${ }^{9}$ Selective deprotection of the primary hydroxyl group in $\mathbf{1 9}$ led to $\mathbf{2 0}$ and further PDC oxidation furnished the sensitive endo-aldehyde 21 (Scheme 4). Addition of vinylmagnesium bromide to 21 gave the diastereomeric mixture $\mathbf{2 2}$ and was as such oxidized with $\mathrm{PDC}$ to enone $\mathbf{2 3}^{9}$ required for effecting the key RCM reaction. Exposure of $\mathbf{2 3}$ to the Grubbs' second generation catalyst ${ }^{11}$ resulted in smooth generation of the desired cycloheptenone moiety and formation of 24. ${ }^{9}$ The stereostructure of $\mathbf{2 4}$ was secured through an $\mathrm{X}$-ray crystal structure determination. ${ }^{12}$

With the availability of the advanced BCD ring intermediate 24, its Diels-Alder reactions with several dienes (1,3-butadiene, isoprene, furan, etc.) were attempted to append the A ring for which the literature precedent 


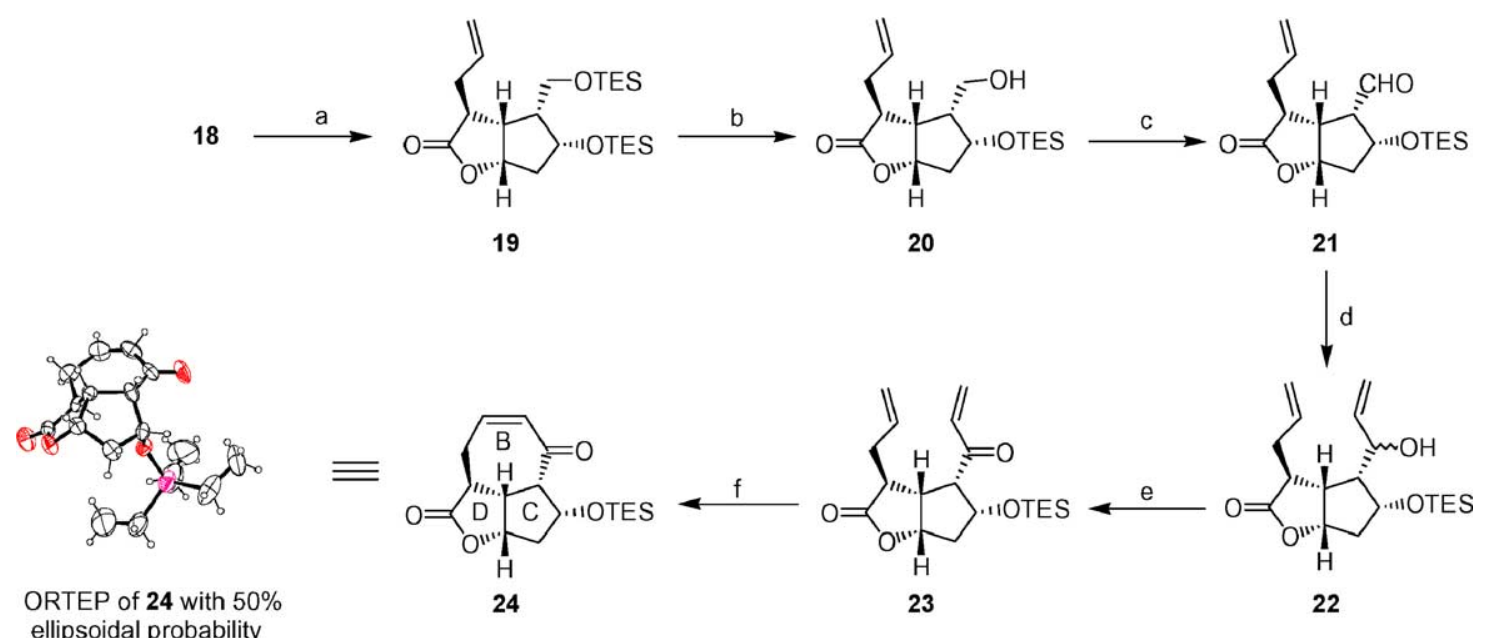

Scheme 4. Reagents and conditions: (a) LHMDS, HMPA, $-78{ }^{\circ} \mathrm{C}, 15 \mathrm{~min}$, allyl bromide, $-78^{\circ} \mathrm{C}, 1.5 \mathrm{~h}, 89 \%$; (b) PPTS, MeOH, $0{ }^{\circ} \mathrm{C}, 4 \mathrm{~h}, 95 \%$; (c) PDC, DCM, $0{ }^{\circ} \mathrm{C}, 3 \mathrm{~h}, 82 \%$ (no column chromatography); (d) $\mathrm{H}_{2} \mathrm{C}=\mathrm{CHMgBr}, \mathrm{THF},-78{ }^{\circ} \mathrm{C}, 30 \mathrm{~min}, 80 \%$; (e) PDC, DCM, $0{ }^{\circ} \mathrm{C}, 4 \mathrm{~h}, 85^{\circ} \%$; (f) Grubbs' II gen. catalyst (10 mol \%), DCM, rt, 5 h, 90\%.

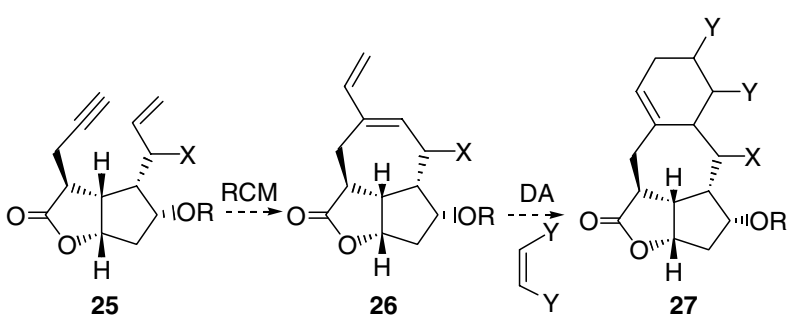

Scheme 5. Enyne metathesis-Diels-Alder strategy.

exists. ${ }^{6}$ However, despite several such attempts under different thermal and catalyzed regimes, success eluded us and this forced us to explore an alternative approach although not successfully. The alternative procedure that we sought to append ring A was through an enyne metathesis ${ }^{13}$ in a precursor such as $\mathbf{2 5}$ to furnish the diene $\mathbf{2 6}$ which was expected to be more amenable to Diels-Alder reaction to deliver ring A as in $\mathbf{2 7}$ (Scheme 5).

Consequently, the TES protected cis-Corey lactone $\mathbf{1 8}$ was propargylated to give $\mathbf{2 8}$ (Scheme 6). ${ }^{9}$ Selective deprotection of the primary hydroxyl group and PDC oxidation led to aldehyde 29. ${ }^{9}$ Addition of vinylmagnesium bromide to $\mathbf{2 9}$ furnished vinyl alcohols $\mathbf{3 0}$, which were further oxidized to enone $\mathbf{3 1}$ to set up the enyne metathesis. Exposure of $\mathbf{3 1}$ to Grubbs' first or second generation catalyst, to our great disappointment, failed to deliver the expected product 32. This called for further tactical adjustments to facilitate the enyne metathesis and efforts along these lines are being pursued.

In summary, we have delineated a synthetic approach towards the novel anti-inflammatory diterpene rameswaralide in which cis-Corey lactone serves as an advanced building block with a tandem RCM reaction and [4+2]cycloaddition as key steps. In this context, a new synthesis of cis-Corey lactone has been devised and it has been further elaborated to the BCD ring core structure of the natural product rameswaralide.

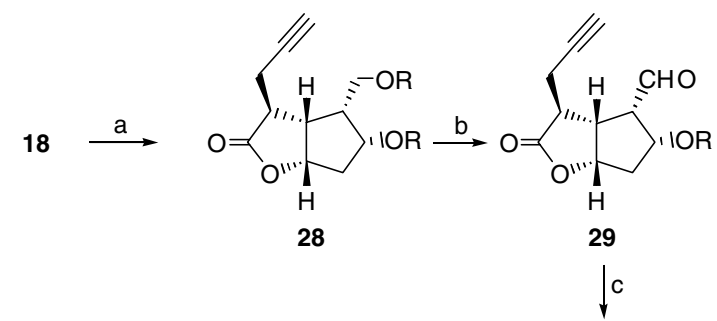

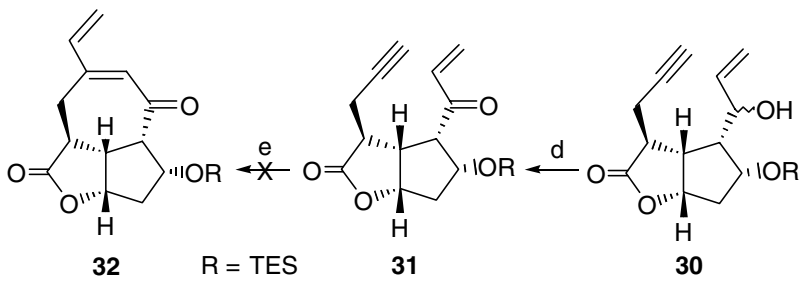

Scheme 6. Reagents and conditions: (a) LHMDS, HMPA, $-78^{\circ} \mathrm{C}$, 15 min, propargyl bromide, $-78^{\circ} \mathrm{C}, 1.5 \mathrm{~h}, 91 \%$; (b) (i) PPTS, $\mathrm{MeOH}$, $0{ }^{\circ} \mathrm{C}, 3 \mathrm{~h}$, quant.; (ii) $\mathrm{PDC}, \mathrm{DCM}, 0{ }^{\circ} \mathrm{C}, 3 \mathrm{~h}, 88 \%$ (no column chromatography); (c) $\mathrm{H}_{2} \mathrm{C}=\mathrm{CHMgBr}$, THF, $-78^{\circ} \mathrm{C}, 30 \mathrm{~min}, 64 \%$; (d) PDC, DCM, $0{ }^{\circ} \mathrm{C}, 4$ h, $80 \%$; (e) Grubbs' I/II gen. catalyst, DCM/ benzene/toluene, $\mathrm{rt} / \mathrm{reflux}$.

\section{Acknowledgements}

This research was supported by the Chemical Biology Unit of JNCASR. We thank Mr. Manabendra Das, for his help with some of the experiments. One of us (S.L.) thanks CSIR and IISc for financial support. Crystallographic data was obtained at the CCD facility at IISc, supported by the Department of Science and Technology, Government of India.

\section{References and notes}

1. Ramesh, P.; Reddy, N. S.; Venkateswarlu, Y.; Reddy, M. V. R.; Faulkner, D. J. Tetrahedron Lett. 1998, 39, 82178220.

2. (a) Reddy, M. V. R.; Lakshman, S.; Rao, A. V. R.; Venkateswarlu, Y.; Rao, J. V. J. Nat. Prod. 1993, 56, 970- 
992; (b) Anjaneyulu, A. S. R.; Sagar, K. S.; Venugopal, M. J. R. V. Tetrahedron 1995, 51, 10997-11010.

3. Faulkner, D. J.; Venkateswarlu, Y. PCT Int. Appl., WO 2000027839, 2000, 22pp, CAN 132:352768.

4. Srikrishna, A.; Dethe, D. H. Org. Lett. 2004, 6, 165.

5. (a) Mehta, G.; Krishnamurthy, N. Tetrahedron Lett. 1987, 28, 5945-5948; (b) Mehta, G.; Krishnamurthy, N.; Karra, S. R. J. Am. Chem. Soc. 1991, 113, 5765-5775; (c) Mehta, G.; Umarye, J. D. Org. Lett. 2002, 4, 1063-1066; (d) Mehta, G.; Umarye, J. D.; Gagliardini, V. Tetrahedron Lett. 2002, 43, 6975-6978; (e) Mehta, G.; Umarye, J. D.; Srinivas, K. Tetrahedron Lett. 2003, 44, 4233-4237; (f) Mehta, G.; Pallavi, K.; Umarye, J. D. Chem. Commun. 2005, 4456-4458.

6. (a) Fringuelli, F.; Pizzo, F.; Taticchi, A.; Halls, T. D. J.; Wenkert, E. J. Org. Chem. 1982, 47, 5056-5065; (b) Fringuelli, F.; Guo, M.; Minuti, L.; Pizzo, F.; Taticchi, A.; Wenkert, E. J. Org. Chem. 1989, 54, 710-712.

7. (a) Brown, E. D.; Clarkson, R.; Leeney, T. J.; Robinson, G. E. J. Chem. Soc., Perkin Trans. 1 1978, 1507-1511; (b) Hwang, S. W.; Adiyaman, M.; Khanapure, S.; Schio, L.; Rokach, J. J. Am. Chem. Soc. 1994, 116, 10829-10830; (c) Hwang, S.-W.; Adiyaman, M.; Khanapure, S. P.; Rokach, J. Tetrahedron Lett. 1996, 37, 779-782; (d) Zanoni, G.; Re, S.; Meriggi, A.; Castronovo, F.; Vidari, G. Tetrahedron: Asymmetry 2001, 12, 1785-1792; (e) Renaud, P.; Vionnet, J.-P. J. Org. Chem. 1993, 58, 5895-5896; (f) Rondot, B.; Durand, T.; Vidal, J.-P.; Girard, J.-P.; Rossi, J.-C. J. Chem. Soc., Perkin Trans. 2 1995, 1589-1594.

8. Mehta, G.; Talukdar, P.; Mohal, N. Tetrahedron Lett. 2001, 42, 7663-7666.

9. All new compounds were characterized on the basis of IR, ${ }^{1} \mathrm{H}$ and ${ }^{13} \mathrm{C}$ NMR and HRMS data. Spectral data for selected compounds: 11: IR (neat): $v_{\max } 3417,1732 \mathrm{~cm}^{-1}$. ${ }^{1} \mathrm{H}$ NMR $\left(300 \mathrm{MHz}, \mathrm{CDCl}_{3}\right): \delta 6.42(1 \mathrm{H}, \mathrm{dd}, J=5.7$, $3 \mathrm{~Hz}), 6.04(1 \mathrm{H}, \mathrm{dd}, J=5.7,3 \mathrm{~Hz}), 5.24(1 \mathrm{H}, \mathrm{td}, J=8.1$, $3.3 \mathrm{~Hz}), 3.48(1 \mathrm{H}, \mathrm{d} 1 / 2 \mathrm{ABq}, J=11.1,7.5 \mathrm{~Hz}), 3.42(1 \mathrm{H}$, $\mathrm{d} 1 / 2 \mathrm{ABq}, J=11.1,8.4 \mathrm{~Hz}), 3.05(1 \mathrm{H}$, br s), $2.76(1 \mathrm{H}, \mathrm{br}$ s), $2.40(1 \mathrm{H}$, br s, $-\mathrm{OH}), 2.23(1 \mathrm{H}, \mathrm{ddd}, J=13.2,8.1$, $3.6 \mathrm{~Hz}), 2.05-1.99(1 \mathrm{H}, \mathrm{m}), 1.98\left(3 \mathrm{H}, \mathrm{s}, \mathrm{OCOCH}_{3}\right), 1.04$ $(1 \mathrm{H}, \mathrm{td}, J=13.2,2.4 \mathrm{~Hz}) .{ }^{13} \mathrm{C}$ NMR $\left(75 \mathrm{MHz} \mathrm{CDCl}_{3}\right): \delta$ $171.5,139.7,133.1,74.4,62.4,61.1\left(\mathrm{CH}_{2}\right), 46.2,42.6,31.6$ $\left(\mathrm{CH}_{2}\right), 21.1$. HRMS (ES): $\mathrm{m} / z$ Calculated for $\mathrm{C}_{10} \mathrm{H}_{14} \mathrm{O}_{3}$ : 205.0841 $[\mathrm{M}+\mathrm{Na}]^{+}$, found: 205.0833. Compound 16: $\mathrm{Mp}$ 153-154 ${ }^{\circ} \mathrm{C}$ (decomposes). IR (KBr): $v_{\max } 3496$, $1770 \mathrm{~cm}^{-1}$. ${ }^{1} \mathrm{H}$ NMR $\left(300 \mathrm{MHz}, \mathrm{CD}_{3} \mathrm{OD}\right): \delta 5.38(1 \mathrm{H}$, $\mathrm{d}, J=7.5 \mathrm{~Hz}), 4.33(2 \mathrm{H}$, br s), $3.88(1 \mathrm{H}, \mathrm{d} 1 / 2 \mathrm{ABq}$, $J=10.8,6.9 \mathrm{~Hz}), 3.75(1 \mathrm{H}, \mathrm{d} 1 / 2 \mathrm{ABq}, J=10.8,7.5 \mathrm{~Hz})$, 3.29-3.19 (1H, m), 2.97-2.88 (1H, m), $2.69(1 \mathrm{H}, \mathrm{d} 1 / 2 \mathrm{ABq}$, $J=18.6,4.8 \mathrm{~Hz}), 2.59(1 \mathrm{H}, \mathrm{d} 1 / 2 \mathrm{ABq}, J=18.6,10.8 \mathrm{~Hz})$. ${ }^{13} \mathrm{C}$ NMR $\left(75 \mathrm{MHz}, \mathrm{CD}_{3} \mathrm{OD}\right): \delta 179.8,94.3,81.2,59.6$ $\left(\mathrm{CH}_{2}\right), 47.0,38.9,33.3,31.1\left(\mathrm{CH}_{2}\right)$. LRMS (70 eV, EI): $\mathrm{m} / z$ $250\left[\mathrm{M}^{+}-\mathrm{CH}_{2} \mathrm{OH}-\mathrm{OH}\right]^{+}, 153,123,95,79$. Analysis: $\mathrm{C}_{8} \mathrm{H}_{11} \mathrm{IO}_{4}$ requires: $\mathrm{C}, 32.24 ; \mathrm{H}, 3.72 \%$. Found: $\mathrm{C}, 32.30$; $\mathrm{H}, 3.58 \%$. Compound 18: IR (neat): $v_{\max } 1779 \mathrm{~cm}^{-1} \cdot{ }^{1} \mathrm{H}$ NMR $\left(300 \mathrm{MHz}, \mathrm{CDCl}_{3}\right): \delta 5.06(1 \mathrm{H}, \mathrm{t}, J=7.5 \mathrm{~Hz}), 4.26$ $(1 \mathrm{H}, \mathrm{t}, J=3.3 \mathrm{~Hz}), 3.86(1 \mathrm{H}, \mathrm{d} 1 / 2 \mathrm{ABq}, J=9.9,6.9 \mathrm{~Hz})$,
$3.73(1 \mathrm{H}, \mathrm{d} 1 / 2 \mathrm{ABq}, J=9.9,8.1 \mathrm{~Hz}), 3.14-3.03(1 \mathrm{H}, \mathrm{m})$, $2.71(1 \mathrm{H}, \mathrm{d} 1 / 2 \mathrm{ABq}, J=18.3,4.8 \mathrm{~Hz}), 2.46(1 \mathrm{H}, \mathrm{d} 1 / 2 \mathrm{ABq}$, $J=18.3,11.7 \mathrm{~Hz}), 2.12(1 \mathrm{H}, 1 / 2 \mathrm{ABq}, J=15 \mathrm{~Hz}), 2.07-$ $1.98(1 \mathrm{H}, \mathrm{m}), 1.83(1 \mathrm{H}, \mathrm{dd} 1 / 2 \mathrm{ABq}, J=15,6.6,3.9 \mathrm{~Hz})$, $0.97-0.91 \quad\left(18 \mathrm{H}, \quad \mathrm{m}, \quad 6 \times \mathrm{CH}_{3}\right), \quad 0.62-0.52(12 \mathrm{H}, \quad \mathrm{m}$, $\left.6 \times \mathrm{CH}_{2}\right) .{ }^{13} \mathrm{C}$ NMR $\left(75 \mathrm{MHz}, \mathrm{CDCl}_{3}\right): \delta$ 178.0, 84.6, 73.5, $59.7\left(\mathrm{CH}_{2}\right), 50.4,42.3\left(\mathrm{CH}_{2}\right), 38.4,30.4\left(\mathrm{CH}_{2}\right), 6.7$ $\left(3 \times \mathrm{CH}_{3}\right), 6.6\left(3 \times \mathrm{CH}_{3}\right), 4.6\left(3 \times \mathrm{CH}_{2}\right), 4.3\left(3 \times \mathrm{CH}_{2}\right)$. HRMS (ES): $m / z$ Calculated for $\mathrm{C}_{20} \mathrm{H}_{40} \mathrm{O}_{4} \mathrm{Si}_{2}: 423.2363$ $[\mathrm{M}+\mathrm{Na}]^{+}$, found: 423.2355 . Compound 23: IR (neat): $v_{\max }$ $1768,1698 \mathrm{~cm}^{-1} .{ }^{1} \mathrm{H}$ NMR $\left(300 \mathrm{MHz}, \mathrm{CDCl}_{3}\right): \delta 6.58$ $(1 \mathrm{H}, \mathrm{d} 1 / 2 \mathrm{ABq}, J=17.4,10.2 \mathrm{~Hz}), 6.31(1 \mathrm{H}, 1 / 2 \mathrm{ABq}$, $J=17.4 \mathrm{~Hz}), \quad 5.87-5.74 \quad(1 \mathrm{H}, \quad \mathrm{m}), \quad 5.76 \quad(1 \mathrm{H}, \quad \mathrm{d}$, $J=10.2 \mathrm{~Hz}), 5.20-5.09(2 \mathrm{H}, \mathrm{m}), 4.92(1 \mathrm{H}, \mathrm{t}, J=6.9 \mathrm{~Hz})$, $4.77(1 \mathrm{H}$, br s), 3.35-3.31 $(1 \mathrm{H}, \mathrm{m}), 3.07-3.01(2 \mathrm{H}, \mathrm{m})$, $2.67-2.63(2 \mathrm{H}, \mathrm{m}), 2.19(1 \mathrm{H}, 1 / 2 \mathrm{ABq}, J=15 \mathrm{~Hz}), 1.98$ $(1 \mathrm{H}, \mathrm{dd} 1 / 2 \mathrm{ABq}, J=15,6.9,3.6 \mathrm{~Hz}), 0.99-0.83(9 \mathrm{H}, \mathrm{m}$, $\left.3 \times \mathrm{CH}_{3}\right), \quad 0.62-0.52 \quad\left(6 \mathrm{H}, \quad \mathrm{m}, \quad 3 \times \mathrm{CH}_{2}\right) .{ }^{13} \mathrm{C} \quad \mathrm{NMR}$ $\left(75 \mathrm{MHz}, \mathrm{CDCl}_{3}\right): \delta 196.8,179.7,134.7,134.3,128.1$ $\left(\mathrm{CH}_{2}\right), 118.5\left(\mathrm{CH}_{2}\right), 81.7,74.4,59.9,43.2,43.1,42.5$ $\left(\mathrm{CH}_{2}\right), 36.6\left(\mathrm{CH}_{2}\right), 6.7\left(3 \times \mathrm{CH}_{3}\right), 4.6\left(2 \times \mathrm{CH}_{2}\right)$. HRMS (ES): $m / z$ Calculated for $\mathrm{C}_{19} \mathrm{H}_{30} \mathrm{O}_{4} \mathrm{Si}: 373.1811[\mathrm{M}+\mathrm{Na}]^{+}$, found: 373.1801 . Compound 24: $\mathrm{Mp} 99-100{ }^{\circ} \mathrm{C}$. IR (KBr) $v_{\max } 1766,1662 \mathrm{~cm}^{-1} .{ }^{1} \mathrm{H}$ NMR $\left(300 \mathrm{MHz}, \mathrm{CDCl}_{3}\right): \delta$ $6.45(1 \mathrm{H}, \mathrm{dd} 1 / 2 \mathrm{ABq}, J=12.9,4.8,3 \mathrm{~Hz}), 6.09(1 \mathrm{H}, \mathrm{d} 1 /$ $2 \mathrm{ABq}, J=12.9,3 \mathrm{~Hz}), 5.07(1 \mathrm{H}, \mathrm{t}, J=7.5 \mathrm{~Hz}), 4.82(1 \mathrm{H}$, $\mathrm{t}, J=6.8 \mathrm{~Hz}), 4.13(1 \mathrm{H}, \mathrm{dt}, J=12,3 \mathrm{~Hz}), 3.04-2.91(3 \mathrm{H}$, $\mathrm{m}), 2.42-2.29(2 \mathrm{H}, \mathrm{m}), 2.11(1 \mathrm{H}, \mathrm{d} 1 / 2 \mathrm{ABq}, \quad J=15$, $1.8 \mathrm{~Hz}), 0.90\left(9 \mathrm{H}, \mathrm{m}, 3 \times \mathrm{CH}_{3}\right), 0.56\left(6 \mathrm{H}, \mathrm{m}, 3 \times \mathrm{CH}_{2}\right)$. ${ }^{13} \mathrm{C} \mathrm{NMR}\left(75 \mathrm{MHz}, \mathrm{CDCl}_{3}\right): \delta 198.6,177.4,142.3,134.3$, 82.0, 75.8, 58.7, 45.3, 42.2, $42.0\left(\mathrm{CH}_{2}\right), 32.7\left(\mathrm{CH}_{2}\right), 6.6$ $\left(3 \times \mathrm{CH}_{3}\right), 4.5\left(3 \times \mathrm{CH}_{2}\right)$. HRMS (ES): $\mathrm{m} / \mathrm{z}$ Calculated for $\mathrm{C}_{17} \mathrm{H}_{26} \mathrm{O}_{4} \mathrm{Si}: 345.1498[\mathrm{M}+\mathrm{Na}]^{+}$, found: 345.1491 . Compound 29: IR (neat): $v_{\max } 2729,1767,1723 \mathrm{~cm}^{-1} \cdot{ }^{1} \mathrm{H}$ NMR $\left(300 \mathrm{MHz}, \mathrm{CDCl}_{3}\right): \delta 9.85(1 \mathrm{H}, \mathrm{s},-\mathrm{CHO}), 5.06(1 \mathrm{H}$, $\mathrm{t}, J=7.5 \mathrm{~Hz}), 4.87(1 \mathrm{H}, \mathrm{t}, J=3.3 \mathrm{~Hz}), 3.33(1 \mathrm{H}, \mathrm{dt}$, $J=8.1,5.4 \mathrm{~Hz}), 3.16(1 \mathrm{H}, \mathrm{q}, J=5.1 \mathrm{~Hz}), 2.89-2.72(3 \mathrm{H}$, $\mathrm{m}), 2.22(1 \mathrm{H}, 1 / 2 \mathrm{ABq}, J=15.3 \mathrm{~Hz}), 2.05-1.94(2 \mathrm{H}, \mathrm{m})$, $0.95-0.90\left(9 \mathrm{H}, \mathrm{m}, 3 \times \mathrm{CH}_{3}\right), 0.63-0.55\left(6 \mathrm{H}, \mathrm{m}, 3 \times \mathrm{CH}_{2}\right)$. ${ }^{13} \mathrm{C}$ NMR $\left(75 \mathrm{MHz}, \mathrm{CDCl}_{3}\right): \delta 200.2,178.1,82.6,80.4$, 73.9, 70.7, 61.0, 43.0, 42.8, 42.1 $\left(\mathrm{CH}_{2}\right), 21.7\left(\mathrm{CH}_{2}\right), 6.6$ $\left(3 \times \mathrm{CH}_{3}\right), 4.5\left(3 \times \mathrm{CH}_{2}\right)$.

10. Corey, E. J.; Weinshenker, N. M.; Schaff, T. K.; Huber, W. J. Am. Chem. Soc. 1969, 91, 5675.

11. Scholl, M.; Ding, S.; Lee, C. W.; Grubbs, R. H. Org. Lett. 1999, 1, 953-956.

12. X-ray data for 24: $\mathrm{C}_{17} \mathrm{H}_{26} \mathrm{O}_{4} \mathrm{Si}, M_{\mathrm{w}}=322.5$, colourless crystal, crystal system: orthorhombic, space group: $P n a 2_{0}$, cell parameters $a=21.6885$ (87) $\AA, b=7.8197$ (31) $\AA$, $c=10.6843$ (43) $\AA, V=1812.03$ (13) $\AA^{3}, Z=4, \rho_{\text {calcd }}=$ $1.86 \mathrm{~g} \mathrm{~cm}^{-3}, F(000)=695.9, \mu=0.144 \mathrm{~mm}^{-1}$. Total no of 1.s. parameters: 202, $R_{1}=0.098$ for 1702 reflections with $F o>4 \operatorname{sig}(F o)$ and 0.156 for all 2792 reflections, $w R_{2}=$ $0.239, \mathrm{GOF}=1.060$, restrained $\mathrm{GOF}=1.060$ for all data (CCDC 281171).

13. Diver, S. T.; Giessert, A. J. Chem. Rev. 2004, 104, 13171382. 